\title{
Detection of Group A Beta-Hemolytic Streptococcus Employing Three Different Detection Methods: Culture, Rapid Antigen Detecting Test, and Molecular Assay
}

\author{
Odimara Santos ${ }^{1}$, Luc Louis Maurice Weckx ${ }^{1}$, \\ Antonio Carlos Campos Pignatari ${ }^{2}$ and \\ Shirley Shizue Nagata Pignatari ${ }^{1}$
}

\author{
Federal University of São Paulo, Division of Pediatric \\ Otolaryngology ${ }^{1}$, Division of Infectious Diseases ${ }^{2}$, São \\ Paulo, SP, Brazil
}

\begin{abstract}
In order to study the prevalence of Group A beta-hemolytic Streptococcus (GABHS) pharyngotonsillitis in our pediatric population and to compare different sampling methods of GABHS detection, oropharyngeal swabs from 50 children with acute pharyngotonsillitis, between 1 and 12 years old, were used simultaneously for culture, molecular assay and rapid GABHS antigen detection tests. All children were clinically examined at the Division of Pediatric Otorhinolaryngology of the Federal University of São Paulo. Diagnostic criteria were based on signs and symptoms, including sore throat, fever and oropharyngeal purulent secretion. Children that had been treated with antibiotics were excluded. Overall, combining the three methods, the prevalence of GABHS was $34 \%$. GABHS was diagnosed in $30 \%$ of the bacterial cultures, in $25 \%$ of the samples tested with the molecular nucleic acid hybridization method and in $26 \%$ of the cases tested with the rapid antigen detection test. There was no significant difference between these three methods.
\end{abstract}

Key Words: Acute pharyngotonsillitis, GABHS, laboratory detection.
Pharyngotonsillitis is one of the most common respiratory diseases in the community, particularly during childhood. Approximately $28 \%$ to $40 \%$ of these infections are estimated to be caused by Group A betahemolytic Streptococcus (GABHS), which is considered the most important etiological pathogen in terms of sequelae and complications [1]. Since streptococcal pharyngotonsillitis can lead to rheumatic disease and its sequelae, and to suppurative complications, it is a potentially serious medical problem, and adequate treatment is imperative.

Despite many attempts to clinically differentiate Streptococcal pharyngotonsillitis from viral infections, so far no method has proven to be reliable [2]. Due to the lack of an adequate clinical basis to diagnose GABHS pharyngotonsillitis, it is important to find an Received on 17 May 2003; revised 30 August 2003. Address for correspondence: Dr. Shirley Shizue Nagata Pignatari Rua dos Otonis 674. Vila Clementino, S Paulo, SP, Brazil. Zip code: 04025001.E-mail: pigna @ terra.com.br

The Brazilian Journal of Infectious Diseases 2003;7(5):297-300 (C) 2003 by The Brazilian Journal of Infectious Diseases and Contexto Publishing. All rights reserved. easy, practical and cost-effective way to provide such a differentiation.

During the last fifty years, bacterial culture has been the main technique used to diagnose GABHS. In 1980, the Center for Disease Control (CDC), estimated that 28 to 36 million throat cultures were performed each year in the USA. Throat culture is still considered the "gold standard" method for GABHS detection, giving positive results in about $90 \%$ to $99 \%$ of the cases [3].

The use of rapid tests to detect GABHS directly from throat swabs has become very popular in many countries. Most of these tests employ latex agglutination or enzyme immunoassay methods to detect the Group A carbohydrate antigen of Streptococcus pyogenes [4-7].

Although molecular hybridization tests are not as popular, they have been shown to have a high level of specificity and sensitivity [8].

To determine the prevalence of GABHS pharyngeal infection, and to find the best way to make this diagnosis, we evaluated children with acute pharyngotonsillitis and compared three different methods of GABHS detection from throat swabs: 
culture in blood-agar, rapid GABHS detection test, and the GABHS nucleic acid hybridization test.

\section{Materials and Methods}

Fifty children with acute pharyngotonsillitis, between 1 and 12 years old were evaluated at the Division of Pediatric Otorhinolaryngology - Federal University of São Paulo, Brazil, from 1998-2001. Diagnostic criteria and selection were based on clinical signs and symptoms, including sore throat, fever, cervical lymphadenitis and purulent oropharyngeal secretions. Parents or legal guardians signed an informed consent.

Oropharyngeal swabs were taken simultaneously for the three detection methods:

1. Culture. Oropharyngeal samples were collected with "Starswab" (Laborclin®), immediately transported to the Microbiology Laboratory and spread on a $5 \%$ blood agar plate (Columbia agar-base, Oxoid), followed by $18-24$ hours incubation at $36^{\circ} \mathrm{C}$ in a $5 \% \mathrm{CO}_{2}$ environment. Biochemical, anti-sera agglutination and optoquin/bacitracin sensitivity tests were performed to identify the GABHS.

2. Samples for the rapid detection test (Test Pack® Strep A - Abbott) were collected in alginated swabs. The tests were all performed "in loco", according to the method provided by the manufacturer. The rapid detection test (Abbott) is based on the detection of the GABHS antigen through an ELISA antigen-AC agglutination reaction.

3. Identification of GABHS by the nucleic acid hybridization test (GEN-PROBE®) INCORPORATED, San Diego, California AccuPACE GROUP A STREPTOCOCCUS IDENTIFICATION TEST) following the manufacturer's specifications. Alginated swabs were inoculated into Tryptic Soy Broth, and incubated at $36^{\circ} \mathrm{C}$ for 18 to 24 hours, in an environment enriched with $\mathrm{CO}_{2}$. Results were obtained by chemiluminescence. Values of 20,000 relative luminescence units (RLU) or higher were considered positive; under 15,000 RLU was considered negative, and between 15,000 and 19,999 was weakly positive.

\section{Results}

The prevalence of Streptococcus pyogenes was $34 \%$ (17 cases) with the combination of the three methods. GABHS was detected in $30 \%$ (15 cases) of the cultures, $26 \%$ (12 cases) by molecular assay tests, and $26 \%$ (13 cases) by the rapid identification test (Table 1).

Considering the culture result as a "gold standard" parameter, the rapid test gave a sensitivity of $73 \%$ (positive cases compared to the total positive cases obtained by culture), and a specificity of $94 \%$ (negative results compared to the total number of negative culture cases), a positive predictive value of $85 \%$, and a negative predictive value of $88 \%$ (Table 2 ).

Molecular tests (acid nucleic hybridization tests) were performed on 47 samples. Concordance of results by using the different methods occurred in $76 \%$ of the cases. In four samples $(8.5 \%)$, the molecular assay was positive, while the culture was negative.

Table 1. Frequency of Group A beta-hemolytic Streptococcus according to the three detection methods

\begin{tabular}{|c|c|c|c|c|c|c|}
\hline \multirow[t]{2}{*}{ Results } & \multicolumn{2}{|c|}{$\begin{array}{c}\text { Rapid test } \\
(n=49)\end{array}$} & \multicolumn{2}{|c|}{$\begin{array}{l}\text { Culture } \\
(n=50)\end{array}$} & \multicolumn{2}{|c|}{$\begin{array}{l}\text { Gene probe } \\
\qquad(\mathrm{n}=47)\end{array}$} \\
\hline & $\mathbf{N}$ & $\%$ & $\mathbf{N}$ & $\%$ & $\mathbf{N}$ & $\%$ \\
\hline Positive & 13 & 26 & 15 & 30 & 12 & 25 \\
\hline Negative & 36 & 73 & 35 & 70 & 35 & 74 \\
\hline
\end{tabular}

Table 2. Diagnostic value of the rapid test

\begin{tabular}{llcc}
\hline & \multicolumn{2}{c}{ Culture } \\
\cline { 3 - 4 } & & Positive & Negative \\
\hline \multirow{2}{*}{ Rapid test } & Positive & 11 & 02 \\
& Negative & 04 & 32 \\
\hline
\end{tabular}




\section{Discussion}

Streptococcal pharyngotonsillitis has been a matter of medical concern over the years, particularly because of its potential for causing serious problems, such as rheumatic fever and suppurative complications. The prevalence of acute pharyngotonsillitis caused by GABHS is approximately $28 \%$ to $40 \%$ worldwide; most are known to be susceptible to penicillin. This percentage also varies from region to region [1].

The prevalence of GABHS in São Paulo, Brazil appears to be similar to the international figures, since overall, GABHS was found in $34 \%$ of the children. These GABHS isolates were also all susceptible to penicillin (data not shown).

The unreliability of the diagnosis based on clinical grounds, has been one of the limiting factors to an adequate management of this disease, especially in developing countries where the rapid detecting tests or even bacterial cultures are not always available. A number of commercial test kits are current available in many countries for the rapid diagnosis of group A streptococcal pharyngotonsillitis, and the most recent are immunoassay-based. Their specificity reaches around $89-99 \%$, and the sensitivity varies between 77 and $98 \%$, considering bacterial culture as a "gold standard" parameter [5]. Most of these tests can be performed "in loco", and the results are available in approximately 15 minutes.

In countries where rapid detection tests are routinely used, a controversy exists regarding whether or not a confirmatory culture is necessary when the results are negative. Although most the doctors do not usually follow this procedure, most of the medical societies have recommended backup cultures [9]. The use of a rapid detection test, plus a bacterial culture for the negative results, is currently considered the most effective clinical strategy, increasing the marginal costs only slightly (it increases the initial costs, but lowers the global cost when considering the prevention of complications of the non-treated rapid test undetected cases) [10].

Combining these three methods, the prevalence of Streptococcus pyogenes in this study was slightly higher than in our previous study, where bacterial culture was the method used. In our study, made in 1996, 18\% of the cultures from 44 children with oropharyngeal infection had GABHS [11].

Although in our current study the molecular assay method was able to detect GABHS in some cases with negative culture results, it was time consuming and expensive. Pokorski et al. (1994) [8], in a study of 767 patients with pharyngitis, the sensitivity of the molecular assay method was $85.7 \%$ and the specificity was, $97.8 \%$, compared to $96.7 \%$ sensitivity and $100 \%$ specificity with bacterial cultures.

Given the international diversity of social and economic conditions, and even the differences of specificity and sensitivity of GABHS detection methods found in several studies, many options of GABHS pharyngotonsillitis management could be considered.

According to a cost-benefit analysis, treating all the patients, without a previous culture or test would prevent $90 \%$ of the potential complications, decreasing the overall costs [10]. On the other hand, there would be more side effects from antibiotics, and their use would be unnecessary in most cases. Whenever an isolated rapid detection test is chosen (with no back up culture), cost-benefit advantages may be significant if the test sensitivity and the followup rate are high [10].

Although several studies have shown that the rapid test, applied alone, does not have sufficient sensitivity to eliminate the need for cultures [7], some researchers have demonstrated that the more recently available rapid tests can be more sensitive than bacterial culture, particularly the immunoassay-based tests, which can give results in a few minutes [6].

Interestingly, bacterial culture has been considered by many as the "gold standard" method of GABHS detection, however according to recent studies, this would be a high cost choice in relation to its effectiveness [10].

Overall, we found the prevalence of GABHS in our population to be similar to international data, around $30 \%$. We also found no significant differences in sensitivity or specificity when comparing the rapid test detection with bacterial culture results. 
Because of the higher costs and the considerable time and labor involved in molecular assay method, we would not recommend it for routine use.

Despite the considerable economic and social diversity existing in our country, where often the rapid test or even bacterial culture techniques are not available, which may lead doctors to diverse management options, including treating the patient based only on clinical assumptions, we believe that objective diagnostic procedures, such as the rapid test or bacterial cultures, should be stimulated.

\section{Acknowledgements}

We thank Mr. Marcel Frederico de Lima Taga from the Dept. of Preventive Medicine, Biostatistical Division, UNIFESP (São Paulo) for his kindness in assisting us with the statistical analyses. We also thank much Dr. Lee Harker; Deputy Director of the Boystown R.C. (Omaha) for his generous help in revising the text. This paper was presented at the American Academy of OtolaryngologyHead and Neck Surgery Meeting held in San Diego, California, USA, (September 22-25, 2002).

\section{References}

1. Pichicheri M.E. Group A Streptococcal tonsillopharyngitis: Cost-effective diagnosis and treatament. Ann Emerg Med 1995;25:390-403.

2. Funamuta J.L, Berkowitz C.D. Applicability of a scoring system in the diagnosis of streptococcal pharyngitis. Clin Pediatr 1983;22:622-9.

3. Mackin M., Hall G., Rutherford I. Three simultaneously obtained throat cultures for beta-hemolytic group A Streptococcus. Pediatr Infect Dis J 1987;6:575-6.

4. Dale J.C., Vetter E.A., Contezac J.M., et al. Evaluation of two rapid antigen assays, BioStar Strep A OIA and Pacific Biotech CARDS OS, and culture for detection of Group A Streptococci in throat swabs. J Clin Microbiolol 1994;32:2698-701.

5. Schager T.A, Hayden G.A, Woods W.A, et al. Optical immunoassay for rapid detection of Group A $\beta$ hemolytic Streptococci. Should culture be replaced? Arch Pediatr Adolesc Med 1996;150: 245-8.

6. Gerber M.A., Tanz R.R., Kabat W., et al. Optical Immunoassay test for Group A $\beta$-hemolytic Streptococcal pharyngitis. An office-bases, multicenter investigation. JAMA 1997;277:899-903.
7. Roe M., Kishiyama C., Davidson K., et al. Comparison of BioStar strep A OIA optical immunoassay, Abbott TestPack Plus Strep A, and culture with selective media for diagnosis of Group A Streptococcal pharyngitis. J Clin Microbiol 1995; 33:1551-3.

8. Pokirski S.J., Vetter E.A, Wollan P.C., Cockerill F.R. Comparasion of gen-probe Group A Streptococcus direct test with culture for diagnosing Streptococcal pharyngitis. J Clin Microbiol 1994;32: 1440-3.

9. Schwartz B., Fries S., Fizgibbon A., Lipman H. Pediatrician's diagnostic approach to pharyngitis and impact of CLIA 1988 on office diagnostic tests. JAMA 1994;271:234-8.

10. Lieu T.A, Fleisher G.R., Schwart J.S. Cost-Effectiveness of rapid latex agglutination testing and throat culture for Streptococcal pharyngitis. Pediatrics 1990;85:24656.

11. Pignatari S.N., Santos O.P., Sader H., et al. Estudo microbiológico de amigdalites agudas em crianças. Anais do XXXIII Congresso Brasileiro de Otorrinolaringologia; IV Congresso Norte-Nordeste de Otorrinolaringologia; 1996. 